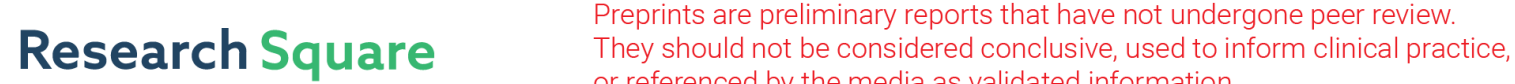 or referenced by the media as validated information. \\ High Expression of HSPA8 is A Favorable Prognostic Factor in Colon Cancer
}

\section{Yang-yang Liu}

The People's Hospital Of Leshan

Xiang-biao He ( $D$ 546036415@qq.com )

The People's Hospital Of Leshan

\section{Research Article}

Keywords: HSPA8, colon adenocarcinoma, immune infiltration, biomarker, prognosis

Posted Date: November 11th, 2021

DOI: https://doi.org/10.21203/rs.3.rs-1059713/v1

License: (c) (i) This work is licensed under a Creative Commons Attribution 4.0 International License. Read Full License 


\section{Abstract}

Background: Colon adenocarcinoma (COAD), a type of colon cancer, is among the most common malignant diseases afflicting people globally. Heat shock protein family, including HSPA8, are involved in tumor progression in numerous human cancers, however the association between HSPA8 and COAD has not been explored.

Methods: RNA sequencing data and clinical characteristics from TCGA and GTEx, as well as protein data from CPTAC were obtained for analysis of HSPA8 expression in COAD. Furthermore, functional enrichment analysis of HSPA8-related differentially expressed genes (DEGs) was performed via GO/KEGG, immune infiltration analysis, and protein-protein interaction (PPI) network. In addition, the clinical significance of HSPA8 was analyzed by Kaplan-Meier Cox regression and prognosis assessment. $\mathrm{R}$ package was employed to analyze incorporated studies.

Results: Compared with normal samples, the expression of HSPA8 increased remarkably in various tumors, including COAD, Moreover, high HSPA8 expression was associated with good prognosis in COAD, as well as subgroups with male, T2-T4 stage, no lymphatic and perineural invasion. $(P<0.05)$. High HSPA8 was a prognostic factor in Cox regression analysis. The diagnostic efficacy of HSPA8 in COAD analyzed by ROC (AUC: 0.889). Furthermore, a total of 3321 DEGs were identified between the high- and the low-expression group, of which 9 genes were up-regulated and 3312 genes were down-regulated.

Conclusions: High HSPA8 expression is a potential biomarker for favorable outcomes in COAD. the results might provide clues and guidance for developing novel diagnosis, targeted therapy and prognosis of HSPA8 in COAD.

\section{Bcakground}

Colon cancer is one of the most common gastrointestinal malignancy with increasing incidence worldwide, and the majority pathologic type is colon adenocarcinoma (COAD). Dietary and lifestyle risk factors, such as high-fat and high-sugar diets, physical inactivity, and obesity, have been related to an increased risk of colon cancer [1]. A clinical need is unmet for improved biomarkers for early diagnosis, prognosis, and risk assessment as well as therapy management for colon cancer patients.

Extracellular vesicles (EVs) are membranous vesicles that are released by eukaryotic cells into the extracellular microenvironment, which include exosomes, microvesicles, and other membrane-encased particles released and taken up by cells as a form of extracellular communication [2]. Chen found that EVs could be employed for detection, prognosis of cancer, and to guide therapy, as well as Sun [4], and several recent studies have shown that EVs perform a critical role in colon cancer [5,6,7]. Many exosome markers, belong to EVs markers have been investigated, including HSPA8, CD9, ALIX, and HSP90AB1. Hoshino A. reported that HSPA8 was the only protein found in $>50 \%$ of 497 normal and cancer associated human and murine-derived samples [8]. 
Heat shock $70 \mathrm{kDa}$ protein 8 (HSPA8), a member of the heat shock protein 70 (HSP70) family, plays an integral role in the protein quality control system, including the folding of newly synthesized proteins, the refolding of misfolded proteins and targeting of proteins for subsequent degradation. HSPA8 has also been found associated with tumor progression in mounting human cancers, such as hepatocellular carcinoma [9], acute myeloid leukemia [10], endometrial carcinoma [11], and usually leads to poor outcomes. However, the role of HSPA8 expression in colon cancer remains poorly understood.

In this way, we aimed to explore the relationship between the expression level of HSPA8 and the prognosis of COAD in our study by using bioinformatics resources. Expression of HSPA8 is elevated significantly in many types of tumors, including COAD, however, unlike other types of malignant tumors, high HSPA8 expression is associated with a good prognosis in COAD. The result indicates that HSPA8 may could be a potential biomarker in the diagnosis, therapy and follow-up of colon cancer.

\section{Results}

\section{High HSPA8 expression in many pan-cancer, including COAD.}

The data of RNA-seq was download from UCSC XENA in TCGA and GTEx formats uniformly processed. The expression level of HSPA8 increased significantly in 21 types of cancer (Figure 1A), colon cancer (COAD) included (Figure 1B), as for paired tumor and adjacent samples, HSPA8 was found clearly high expressed as well (Figure 1C), the differences above all was statistically significant $(P<0.001)$.

To further validate the expression of protein, we explored the patterns of HSPA8 in COAD by Human Protein Atlas and UALCAN. As was shown in Figure 1D, HSPA8 protein was not detected in normal colon tissues, while medium expressions were observed in COAD samples. We next analyzed the relationship of HSPA8 protein expression in colon cancer between normal and primary tumor by UALCAN (Figure 2A), and found that protein expression of tumor group surpassed normal tissues obviously $(P<0.001)$.

\section{Clinical Characteristics}

The clinical data of 478 patients of COAD were obtained from TCGA, including age, race, gender, primary therapy outcome, CEA level, TNM stage, pathological stage, perineural invasion, lymphatic invasion (Table 1). Among them, HSPA8 expression was 50\% high and 50\% low in 478 cases, according the cut-off valve, which was the median HSPA8 expression. Correlation analysis elucidated that HSPA8 expression was significantly correlated with age $(P=0.050)$, race $(P=0.035) \mathrm{N}$ stage $(P<0.001)$, M stage $(P=0.027)$, pathological stage $(P=0.002)$, OS event $(P=0.045)$.

Table 1 clinical characteristic of the COAD patients form TCGA. 


\begin{tabular}{|c|c|c|c|}
\hline Characteristic & Low expression of HSPA8 & High expression of HSPA8 & $p$ \\
\hline $\mathrm{n}$ & 239 & 239 & \\
\hline T stage, n (\%) & & & 0.150 \\
\hline T1 & $7(1.5 \%)$ & $4(0.8 \%)$ & \\
\hline $\mathrm{T} 2$ & $37(7.8 \%)$ & $46(9.6 \%)$ & \\
\hline T3 & $157(32.9 \%)$ & $166(34.8 \%)$ & \\
\hline T4 & $37(7.8 \%)$ & $23(4.8 \%)$ & \\
\hline N stage, n (\%) & & & $<0.001$ \\
\hline NO & $121(25.3 \%)$ & $163(34.1 \%)$ & \\
\hline $\mathrm{N} 1$ & $66(13.8 \%)$ & $42(8.8 \%)$ & \\
\hline N2 & $52(10.9 \%)$ & $34(7.1 \%)$ & \\
\hline M stage, n (\%) & & & 0.027 \\
\hline MO & $162(39 \%)$ & $187(45.1 \%)$ & \\
\hline M1 & $41(9.9 \%)$ & $25(6 \%)$ & \\
\hline Pathologic stage, n (\%) & & & 0.002 \\
\hline Stage I & $40(8.6 \%)$ & $41(8.8 \%)$ & \\
\hline Stage II & $76(16.3 \%)$ & $111(23.8 \%)$ & \\
\hline Stage III & $78(16.7 \%)$ & $55(11.8 \%)$ & \\
\hline Stage IV & $41(8.8 \%)$ & $25(5.4 \%)$ & \\
\hline Gender, n (\%) & & & 0.521 \\
\hline Female & $109(22.8 \%)$ & $117(24.5 \%)$ & \\
\hline Male & $130(27.2 \%)$ & $122(25.5 \%)$ & \\
\hline Race, n (\%) & & & 0.035 \\
\hline Asian & $2(0.7 \%)$ & $9(2.9 \%)$ & \\
\hline Black or African American & $38(12.4 \%)$ & $25(8.2 \%)$ & \\
\hline White & $122(39.9 \%)$ & $110(35.9 \%)$ & \\
\hline Age, $n(\%)$ & & & 0.050 \\
\hline$<=65$ & $108(22.6 \%)$ & $86(18 \%)$ & \\
\hline$>65$ & $131(27.4 \%)$ & $153(32 \%)$ & \\
\hline
\end{tabular}




\begin{tabular}{|llll|}
\hline Characteristic & Low expression of HSPA8 & High expression of HSPA8 & $p$ \\
\hline CEA level, $\mathrm{n}(\%)$ & & & 0.101 \\
\hline$<=5$ & $91(30 \%)$ & $105(34.7 \%)$ & \\
$>5$ & $61(20.1 \%)$ & $46(15.2 \%)$ & 0.289 \\
\hline Perineural invasion, $\mathrm{n}(\%)$ & & $67(37 \%)$ & \\
NO & $68(37.6 \%)$ & $18(9.9 \%)$ & 0.051 \\
YES & $28(15.5 \%)$ & & \\
\hline Lymphatic invasion, $\mathrm{n}(\%)$ & & $144(33.2 \%)$ & 0.045 \\
\hline NO & $122(28.1 \%)$ & $74(17.1 \%)$ & \\
YES & $94(21.7 \%)$ & & \\
\hline OS event, $\mathrm{n}(\%)$ & & $197(41.2 \%)$ & \\
\hline Alive & $178(37.2 \%)$ & $42(8.8 \%)$ & \\
\hline Dead & $61(12.8 \%)$ & & \\
\hline
\end{tabular}

To further verify the correlation between COAD clinicopathological factors and the HSPA8 high-low dichotomy, logistic analysis was performed in this study. As a result, high HSPA8 expression showed a significant negative correlation with $\mathrm{N}$ stage (N1\&N2) (odd ratio, $0.478 ; P<0.001$ ), M stage (M1) (odd ratio, 0.528; $P=0.021$ ), pathologic stage (Stage III \& Stage IV) (odd ratio, $0.513 ; P<0.001$ ), whereas positively correlated with age $(>65)$ (odd ratio, $1.467 ; P=0.041$ ) (Table 2). ROC curve analysis was adapted to evaluated the potential valve of HSPA8 in differentiating patients from population, the AUC was 0.889 , the result showed that HSPA8 was a potential biomarker for the diagnosis of COAD (Figure 2B). Moreover, the Wilcoxon rank sum test was employed to contrast the HSPA8 expression in patients with distinct clinicopathological characters. As a result, the expression of HSPA8 was obviously negative associated with $\mathrm{N}$ stage $(P<0.001)$, $\mathrm{M}$ stage $(P=0.013)$, pathologic stage $(P<0.001)$, lymphatic invasion $(P=0.03)$ (Figure 2C-2F).

Table 2 Univariate regression analysis of HSPA8 and other clinical characteristics with OS in COAD 


\begin{tabular}{|c|c|c|c|}
\hline Characteristics & Total(N) & Odds Ratio (OR) & $P$ value \\
\hline T stage (T3\&T4 vs. T1\&T2) & 477 & $\begin{array}{l}0.857(0.544- \\
1.347)\end{array}$ & 0.504 \\
\hline N stage (N1\&N2 vs. N0) & 478 & $\begin{array}{l}0.478(0.329- \\
0.692)\end{array}$ & $<0.001$ \\
\hline M stage (M1 vs. M0) & 415 & $\begin{array}{l}0.528(0.305- \\
0.901)\end{array}$ & 0.021 \\
\hline $\begin{array}{l}\text { Pathologic stage (Stage III\&Stage IV vs. Stage I\&Stage } \\
\text { II) }\end{array}$ & 467 & $\begin{array}{l}0.513(0.353- \\
0.743)\end{array}$ & $<0.001$ \\
\hline Age (>65 vs. <=65) & 478 & $\begin{array}{l}1.467(1.017- \\
2.120)\end{array}$ & 0.041 \\
\hline Perineural invasion (YES vs. NO) & 181 & $\begin{array}{l}0.652(0.326- \\
1.281)\end{array}$ & 0.219 \\
\hline CEA level (>5 vs. $<=5$ ) & 303 & $\begin{array}{l}0.654(0.405- \\
1.049)\end{array}$ & 0.079 \\
\hline
\end{tabular}

\section{Performance of DEGs in COAD samples with high- and low expressed HSPA8}

The gene expression profiles were divided into high- and low-expression groups according to the criteria of median HSPA8 mRNA expression (Additional file 1: S1). analyzed for differences in the median mRNA expression. A total of 3321 DEGs from gene expression RNA-seq-HTSeq-Counts, including 9 up-regulated and 3312 down-regulated, were identified statistically significant between the two groups (llog fold change $(\log F C) \mid>1.5, P<0.05)$ (Figure $3 A)$. The top five up-regulated DEGs and top five down-regulated DEGs were shown by the heat map (Figure 3B).

\section{GO/KEGG functional enrichment analysis and PPI network construction}

In association with functional enrichment analysis via GO/KEGG, the correlation with the biological process (BP) consisted of sensory perception of bitter taste and corresponding detection of chemical stimulus involved; cellular component (CC) included nuclear nucleosome and muscle myosin complex; molecular function (MF) included bitter taste receptor activity, taste receptor activity; KEGG included taste transduction, olfactory transduction (Figure 4A, 4B).

We also employed STRING to explore the PPI network of HSPA8 protein to analysis the interaction in COAD, the top 10 gene names and corresponding protein, as well as the annotations and score are shown in Figure 5A, 5B. Moreover, with a threshold of 0.4, the network of HSPA8 and its potential co-expressed genes in HSPA8-related DEGs was constructed by SRING, a total of 114 protein coding were screened out with the threshold of $\mid \log$ fold change (log FC) $\mid>1.5, P<0.05$ (Figure 5C) (Additional file 1: S2).

\section{Immune infiltration analysis in COAD}


Spearman correlation analysis demonstrated that the HSPA8 expression level in the COAD tumor microenvironment was relevant to the immune infiltration quantified by SSGSEA, the expression level of HSPA8 had clearly positive correlation with infiltrating level of Th2 cells, but negative correlation with Plasmacytoid DC (Figure 6).

\section{The expression of HSPA8 affected the prognosis of COAD in patients with different clinicopathological status}

Kaplan-Meier survival analysis shown that high HSPA8 expression in COAD patients was significantly associated with the longer overall survival (OS) (hazard ratio [HR], $0.66(0.45-0.98) ; P=0.041)$ (Figure 7A). It also presented that high expression of HSPA8 correlated with good prognosis in the subgroups, including male ( $P=0.038)$, high T stage (T2-4) $(P=0.017)$, no lymphatic invasion $(P=0.035)$, no perineural invasion $(P=0.035)$ (Figure 7B-7E).

\section{Discussion}

In addition to cancer genetic, aberrant epigenetic regulation has also been reported to participate the development and progression of many types of cancer, including COAD [25]. Heat shock protein (HSP) family members, as components of epigenetic regulation complexes, are important in the development of colorectal cancer [26]. The distinct roles of HSP family members in COAD, however, remained unclear. In our study, the expression of mRNA and protein, and prognostic values of HSPA8 in COAD were explored.

HSPA8, also known as heat shock cognate 70 (HSC70), belongs to heat shock 70kDa protein family, which were notably produced under stress conditions. HSPA8, the major family member is critical for cells to be healthy and function normally [27-30], as well as plays an essential role in many biological tumor processes [31, 32], consisting of cell proliferation, autophagy, immune infiltration. Several studies have elucidated that High HSPA8 expression predicted adverse outcomes of hepatocellular carcinoma [9], acute myeloid leukemia [10], endometrial carcinoma [11]. However, little has been reported about the prognostic value of HSPA8 in COAD, and the clinicopathologic implications of HSPA8 expression in COAD have not been thoroughly studied.

In this study, we found that, comparing to normal cohort, the mRNA and protein expression levels of HSPA8 increased significantly in COAD samples, and the high HSPA8 expression levels were associated with low $\mathrm{N}$ and $\mathrm{M}$ stage, low pathologic stage, and good overall survive. Via GO/KEGG functional enrichment analysis and PPI network construction, we found that HSPA8 regulates tumor related genes, such as STUB1, BAG family, HSP family, MYH family. The results might indicate that HSPA8 was a tumor suppressor gene.

Exosomes, which belong to extracellular vesicles (EVs) and enrich in nucleic acids, lipids, and proteins [33], play important roles in cell-to-cell communication during the tumor progression, immune response, neuron survival and many other contexts [34]. As a traditional exosome marker, HSPA8 has been analyzed in pancreatic cancer, lung cancer [8] and diabetic kidney disease [35], breast cancer [36]. Little is 
known about the relationship between HSPA8 and exosomes in COAD. In present study, HSPA8 had a strong interaction with HSP90AA1, an exosomal protein that implicated in maintenance and cellular adhesion for colorectal cancer cells via integrin-mediated cell adhesion pathway [37]. However further studies were need to confirm the result and explore the potential regulatory mechanism of HSPA8.

In immune cell infiltration analysis, high HSPA8 expression was positively associated with the majority of immune cells in COAD samples, including Th2 cells, Dendritic cells, Macrophages, Neutrophils, Dysbiosis is known and implicated in the growth and progression of COAD, and immune responses play indispensable roles in the procedure $[38,39]$. Therefore, HSPA8 could regulate immune cells and immune responses, and further affecting the progression of colon cancer, but the details of the immunoregulatory mechanisms in COAD still deserve further exploration.

There were some limitations in our study. First, although high HSPA8 expression was an independent prognostic factor for longer OS of colon cancer patients, all the data was retrieved from online databases, further studies that consist of extensive functional in vitro and in vivo work, and larger sample sizes were needed to validate our finding. Second, the retrospective nature of our study was a significant limitation, and further prospective investigation designed trials were warranted. Lastly, we did not explore the potential mechanisms in depth of HSPA8 in COAD, future studies to investigate the potential diagnostic and therapeutic values of HSPA8 in COAD waited to be fully elucidated.

\section{Conclusion}

In summary, the present study demonstrated that the mRNA and protein expression levels of HSPA8 markedly elevated in colon cancer samples compared with normal tissue, and over expression of HSPA8 was found significant associated with lower clinical cancer stages and pathologic grades of COAD. Moreover, high HSPA8 expression predicts favorable outcomes in COAD, which indicated that HSPA8 might be a promising biomarker with diagnostic or therapeutic value, and provide novel target for therapeutics. Therefore, the results above might provide clues and guidance for developing novel diagnosis, targeted therapy and prognosis of HSPA8 in colon cancer.

\section{Materials And Methods}

\section{Data source and Bioinformatics Analysis}

The Cancer Genome Atlas (TCGA) is an open and free data portal of largescale genomics project, provides a comprehensive clinic and pathological information of 33 types of cancer for researchers and scholars. In our study, the pan-cancer RNA-seq data of TCGA and GTEx with toil processed were downloaded from UCSC XENA [12,13]. And data of COAD patients were collected from TCGA, including 308 normal samples from GTEx, 41 para-carcinoma tissues and 290 COAD tissues from TCGA. Clinical data, including gender, age, weight, primary therapy outcome, CEA level, perineural invasion, lymphatic invasion, histologic grade and pathologic stage were summarized. 
UALCAN is a comprehensive, user-friendly, and interactive web resource for analyzing cancer OMICS data from TCGA, MET500 and CPTAC, and has been now visited over 700,000 times by researchers from over 100 countries and cited more than 1900 times [14]. The Clinical Proteomic Tumor Analysis Consortium (CPTAC), now provides protein expression analysis, which for colon cancer is accessible [15]. The Human Protein Atlas is a program established in 2003, and the data in the knowledge resource is open and free access to allow scholars to obtain the data for exploration of the human proteome (Version: 20.1). Direct comparison of protein expression of HSPA8 between human normal and COAD tissues was performed by immunohistochemistry image in this study.

\section{Differentially expressed gene (DEG) analysis}

The ggplot2 R (3.3.3 vision) package was applied to compare the data of low- and high-expression of HSPA8 (cut-off value of 50\%) in COAD samples to identify DEGs [16]. The top 10 DEGs were exhibited by heat map.

\section{Functional enrichment analysis}

The DEGs, which were identified and filtered with threshold for $\| \log$ fold change (log FC) $\mid>1.5$ and $P<$ 0.05 were used to functional enrichment analysis. Kyoto Encyclopedia of Genes and Genomes (KEGG) pathway analysis, and Gene Ontology (GO) functional analysis including cellular component (CC), molecular function (MF), and biological process (BP), were performed by using the clusterProfiler package (3.14.3), GO plot package (1.0.2) and ggplot2 (3.3.3) in R [17].

\section{Immune infiltration analysis}

Immune infiltration analysis of HSPA8 was conducted using GSVA package by sSGSEA in R (3.6.3) [18]. Spearman correction was used to analyze the correlation between HSPA8 and the enrichment scores of 24 types of immune cells [19]. Independent-samples T test was used to analyze the enrichment scores of high- and low-expression groups of HSPA8.

\section{Protein-protein interaction (PPI) network}

The PPI network of DEGs was predicted using the Search Tool for the Retrieval of Interacting Genes (STRING) database, and the cut-off criterion was 0.4 [20]. The PPI network was mapped using Cytoscape (version 3.9.0) [21, 22].

\section{Prognosis analysis with multiple modes}

Receiver operating characteristic (ROC) and Kaplan-Meier survival curves were employed to analyze the diagnostic and prognostic values of HSPA8 in COAD patients [12]. Secondly, univariate regression analyses were used to elucidate the association between HSPA8 expression and overall survival (OS) rates [23]. 


\section{Statistical analysis}

All statistical analyses and graphs were analyzed and displayed by R (3.6.3) [24]. The expression of HSPA8 in unpaired samples was analyzed by Wilcoxon rank sum test, with Wilcoxon signed-rank test used in paired samples. Logistic regression analysis was used to evaluate the relationship between clinical characteristics and expression of HSPA8. Kaplan-Meier method were used to evaluate the prognostic factors. Univariate Cox analysis was adopted to compare the impact of HSPA8 expression on survival comparing with other clinical features. The cut-off value was the median HSPA8 expression. In all tests, $\mathrm{P}$ value $<0.05$ was considered statistically significant. Moreover, ROC analysis was performed on the pROC package to assess the effectiveness of the expression of HSPA8.

\section{Declarations}

\section{Acknowledgements}

The authors gratefully acknowledge the Cancer Genome Atlas (TCGA) and Genotype-Tissue Expression (GTEx) database, the Clinical Proteomic Tumor Analysis Consortium (CPTAC) database, Search Tool for the Retrieval of Interacting Genes (STRING) database and Human Protein Atlas which made the data available.

\section{Authors' contributions}

This research was conducted in collaboration with all authors. YL performed the data curation and analysis. $\mathrm{XH}$ analyzed and interpreted the results. $\mathrm{YL}$ and $\mathrm{XH}$ drafted and reviewed the manuscript. All authors read and approved the final manuscript.

\section{Funding}

No funding.

\section{Availability of data and materials}

The raw data of this study are download from the UCSC XENA ( https://xenabrowser.net/datapages/ ), which are publicly available databases.

\section{Declarations}

Ethics approval and consent to participate

Not necessary.

\section{Consent for publication}

Not applicable. 


\section{Competing interests}

The authors declare that they have no competing interests.

\section{References}

1. Murphy, G., Cross, A. J., Dawsey, S. M., Stanczyk, F. Z., Kamangar, F., Weinstein, S. J., Taylor, P. R., Männistö, S., Albanes, D., Abnet, C. C., \& Freedman, N. D. (2018). Serum ghrelin is associated with risk of colorectal adenocarcinomas in the ATBC study. Gut, 67(9), 1646-1651.

2. Antoury, L., Hu, N., Balaj, L., Das, S., Georghiou, S., Darras, B., Clark, T., Breakefield, X. O., \& Wheeler, T. M. (2018). Analysis of extracellular mRNA in human urine reveals splice variant biomarkers of muscular dystrophies. Nature communications, 9(1), 3906.

3. Chen IH, Xue L, Hsu CC, Paez JS, Pan L, Andaluz H, Wendt MK, Iliuk AB, Zhu JK, Tao WA. Phosphoproteins in extracellular vesicles as candidate markers for breast cancer. Proc Natl Acad Sci U S A. 2017 Mar 21;114(12):3175-3180.

4. Sun YZ, Ruan JS, Jiang ZS, Wang L, Wang SM. Extracellular Vesicles: A New Perspective in Tumor Therapy. Biomed Res Int. 2018:2687954.

5. Lee CH, Im EJ, Moon PG, Baek MC. Discovery of a diagnostic biomarker for colon cancer through proteomic profiling of small extracellular vesicles. BMC Cancer. 2018;18(1):1058.

6. Abdouh M, Floris M, Gao ZH, Arena V, Arena M, Arena GO. Colorectal cancer-derived extracellular vesicles induce transformation of fibroblasts into colon carcinoma cells. J Exp Clin Cancer Res. 2019;38(1):257.

7. Zhong, M. E., Chen, Y., Xiao, Y., Xu, L., Zhang, G., Lu, J., Qiu, H., Ge, W., \& Wu, B. (2019). Serum extracellular vesicles contain SPARC and LRG1 as biomarkers of colon cancer and differ by tumour primary location. EBioMedicine, 50, 211-223.

8. Hoshino A, Kim HS, Bojmar L, Gyan KE, Cioffi M, Hernandez J, Zambirinis CP, Rodrigues G, Molina H, Heissel S, Mark MT, Steiner L, Benito-Martin A, Lucotti S, Di Giannatale A, Offer K, Nakajima M, Williams C, Nogués L, Pelissier Vatter FA, Hashimoto A, Davies AE, Freitas D, Kenific CM, Ararso Y, Buehring W, Lauritzen P, Ogitani Y, Sugiura K, Takahashi N, Alečković M, Bailey KA, Jolissant JS, Wang H, Harris A, Schaeffer LM, García-Santos G, Posner Z, Balachandran VP, Khakoo Y, Raju GP, Scherz A, Sagi I, Scherz-Shouval R, Yarden Y, Oren M, Malladi M, Petriccione M, De Braganca KC, Donzelli M, Fischer C, Vitolano S, Wright GP, Ganshaw L, Marrano M, Ahmed A, DeStefano J, Danzer E, Roehrl MHA, Lacayo NJ, Vincent TC, Weiser MR, Brady MS, Meyers PA, Wexler LH, Ambati SR, Chou AJ, Slotkin EK, Modak S, Roberts SS, Basu EM, Diolaiti D, Krantz BA, Cardoso F, Simpson AL, Berger M, Rudin CM, Simeone DM, Jain M, Ghajar CM, Batra SK, Stanger BZ, Bui J, Brown KA, Rajasekhar VK, Healey JH, de Sousa M, Kramer K, Sheth S, Baisch J, Pascual V, Heaton TE, La Quaglia MP, Pisapia DJ, Schwartz R, Zhang H, Liu Y, Shukla A, Blavier L, DeClerck YA, LaBarge M, Bissell MJ, Caffrey TC, Grandgenett PM, Hollingsworth MA, Bromberg J, Costa-Silva B, Peinado H, Kang Y, Garcia 
BA, O'Reilly EM, Kelsen D, Trippett TM, Jones DR, Matei IR, Jarnagin WR, Lyden D. Extracellular Vesicle and Particle Biomarkers Define Multiple Human Cancers. Cell. 2020;182(4):1044-1061.e18.

9. Gao, Z., Ye, X., Bordeaux, A., Hettich, S., Lin, S., Han, F., \& Jia, Y. (2021). miR-26b regulates cell proliferation and apoptosis of CD117+CD44+ ovarian cancer stem cells by targeting PTEN. European journal of histochemistry: EJH, 65(1), 3186.

10. Li J, Ge Z. High HSPA8 expression predicts adverse outcomes of acute myeloid leukemia. BMC Cancer. 2021;21(1):475.

11. Shan N, Zhou W, Zhang S, Zhang Y. Identification of HSPA8 as a candidate biomarker for endometrial carcinoma by using iTRAQ-based proteomic analysis. Onco Targets Ther. 2016; 9: 21692179.

12. Vivian, J., Rao, A. A., Nothaft, F. A., Ketchum, C., Armstrong, J., Novak, A., Pfeil, J., Narkizian, J., Deran, A. D., Musselman-Brown, A., Schmidt, H., Amstutz, P., Craft, B., Goldman, M., Rosenbloom, K., Cline, M., O'Connor, B., Hanna, M., Birger, C., Kent, W. J., ... Paten, B. (2017). Toil enables reproducible, open source, big biomedical data analyses. Nature biotechnology, 35(4), 314-316.

13. Goldman, M. J., Craft, B., Hastie, M., Repečka, K., McDade, F., Kamath, A., Banerjee, A., Luo, Y., Rogers, D., Brooks, A. N., Zhu, J., \& Haussler, D. (2020). Visualizing and interpreting cancer genomics data via the Xena platform. Nature biotechnology, 38(6), 675-678.

14. Chandrashekar, D. S., Bashel, B., Balasubramanya, S., Creighton, C. J., Ponce-Rodriguez, I., Chakravarthi, B., \& Varambally, S. (2017). UALCAN: A Portal for Facilitating Tumor Subgroup Gene Expression and Survival Analyses. Neoplasia (New York, N.Y.), 19(8), 649-658.

15. Chen, F., Chandrashekar, D. S., Varambally, S., \& Creighton, C. J. (2019). Pan-cancer molecular subtypes revealed by mass-spectrometry-based proteomic characterization of more than 500 human cancers. Nature communications, 10(1), 5679.

16. Love, M.I., Huber, W. \& Anders, S. Moderated estimation of fold change and dispersion for RNA-seq data with DESeq2. Genome Biol 15, 550 (2014).

17. Yu G, Wang LG, Han Y, He QY. clusterProfiler: an R package for comparing biological themes among gene clusters. OMICS. 2012 May;16(5):284-7.

18. Hänzelmann, Sonja, Robert Castelo, and Justin Guinney. GSVA: gene set variation analysis for microarray and RNA-seq data. BMC bioinformatics 14.1 (2013): 1-15.

19. Bindea, Gabriela. Spatiotemporal dynamics of intratumoral immune cells reveal the immune landscape in human cancer. Immunity 39.4. (2013): 782-795.

20. Szklarczyk, D., Gable, A. L., Lyon, D., Junge, A., Wyder, S., Huerta-Cepas, J., Simonovic, M., Doncheva, N. T., Morris, J. H., Bork, P., Jensen, L. J., \& Mering, C. V. (2019). STRING v11: protein-protein association networks with increased coverage, supporting functional discovery in genome-wide experimental datasets. Nucleic acids research, 47(D1), D607-D613.

21. Demchak, B., Hull, T., Reich, M., Liefeld, T., Smoot, M., Ideker, T., \& Mesirov, J. P. (2014). Cytoscape: the network visualization tool for GenomeSpace workflows. F1000Research, 3, 151. 
22. Bandettini WP, Kellman P, Mancini C, Booker OJ, Vasu S, Leung SW, Wilson JR, Shanbhag SM, Chen MY, Arai AE. MultiContrast Delayed Enhancement (MCODE) improves detection of subendocardial myocardial infarction by late gadolinium enhancement cardiovascular magnetic resonance: a clinical validation study. J Cardiovasc Magn Reson. 2012 Nov 30;14(1):83.

23. Liu, J., Lichtenberg, T., Hoadley, K. A., Poisson, L. M., Lazar, A. J., Cherniack, A. D., Kovatich, A. J., Benz, C. C., Levine, D. A., Lee, A. V., Omberg, L., Wolf, D. M., Shriver, C. D., Thorsson, V., Cancer Genome Atlas Research Network, \& Hu, H. (2018). An Integrated TCGA Pan-Cancer Clinical Data Resource to Drive High-Quality Survival Outcome Analytics. Cell, 173(2), 400-416.e11.

24. Isidro-Sánchez J, Akdemir D, Montilla-Bascón G. Genome-Wide Association Analysis Using R. Methods Mol Biol. 2017;1536:189-207.

25. Ning, G., Huang, Y. L., Zhen, L. M., Xu, W. X., Jiao, Q., Yang, F. J., Wu, L. N., Zheng, Y. Y., Song, J., Wang, Y. S., Xie, C., \& Peng, L. Transcriptional expressions of Chromobox 1/2/3/6/8 as independent indicators for survivals in hepatocellular carcinoma patients. Aging, 10(11), $3450-3473$.

26. Giaginis C, Daskalopoulou SS, Vgenopoulou S, Sfiniadakis I, Kouraklis G, Theocharis SE. Heat Shock Protein-27, -60 and -90 expression in gastric cancer: association with clinicopathological variables and patient survival. BMC Gastroenterol. 2009 Feb 9;9:14.

27. Bonam SR, Ruff M, Muller S. HSPA8/HSC70 in Immune Disorders: A Molecular Rheostat that Adjusts Chaperone-Mediated Autophagy Substrates. Cells. 2019;8(8):849.

28. Sirtori R, Riva C, Ferrarese C, Sala G. HSPA8 knock-down induces the accumulation of neurodegenerative disorder-associated proteins. Neurosci Lett. 2020;736:135272.

29. Wang F, Bonam SR, Schall N, Kuhn L, Hammann P, Chaloin O, Madinier JB, Briand JP, Page N, Muller S. Blocking nuclear export of HSPA8 after heat shock stress severely alters cell survival. Sci Rep. 2018 Nov 14;8(1):16820.

30. Zhang K, Zhang H, Wang F, Gao S, Sun C. HSPA8 Is Identified as a Novel Regulator of Hypertensive Disorders in Pregnancy by Modulating the $\beta$-Arrestin1/A1AR Axis. Reprod Sci. 2021;10.1007/s43032021-00719-8.

31. Nirdé P, Derocq D, Maynadier M, Chambon M, Basile I, Gary-Bobo M, Garcia M. Heat shock cognate 70 protein secretion as a new growth arrest signal for cancer cells. Oncogene. 2010 Jan 7;29(1):11727.

32. Stricher F, Macri C, Ruff M, Muller S. HSPA8/HSC70 chaperone protein: structure, function, and chemical targeting. Autophagy. 2013;9(12):1937-1954.

33. O'Driscoll L. Expanding on exosomes and ectosomes in cancer. N Engl J Med. 2015; 372(24): 23592362.

34. Villarroya-Beltri C, Baixauli F, Mittelbrunn M, Fernández-Delgado I, Torralba D, Moreno-Gonzalo O, Baldanta S, Enrich C, Guerra S, Sánchez-Madrid F. ISGylation controls exosome secretion by promoting lysosomal degradation of MVB proteins. Nat Commun. 2016 Nov 24; 7:13588.

35. Wen J, Ma Z, Livingston MJ, Zhang W, Yuan Y, Guo C, Liu Y, Fu P, Dong Z. Decreased secretion and profibrotic activity of tubular exosomes in diabetic kidney disease. Am J Physiol Renal Physiol. 2020 
Oct 1;319(4): F664-F673.

36. Chen J, Liu C, Cen J, Liang T, Xue J, Zeng H, Zhang Z, Xu G, Yu C, Lu Z, Wang Z, Jiang J, Zhan X, Zeng J. KEGG-expressed genes and pathways in triple negative breast cancer: Protocol for a systematic review and data mining. Medicine (Baltimore). 2020 May;99(18):e19986.

37. Chen $Y, X i e Y, X u L$, Zhan S, Xiao Y, Gao Y, Wu B, Ge W. Protein content and functional characteristics of serum-purified exosomes from patients with colorectal cancer revealed by quantitative proteomics. Int J Cancer. 2017;140(4):900-913.

38. Belghasem M, Roth D, Richards S, Napolene MA, Walker J, Yin W, Arinze N, Lyle C, Spencer C, Francis JM, Thompson C, Andry C, Whelan SA, Lee N, Ravid K, Chitalia VC. Metabolites in a mouse cancer model enhance venous thrombogenicity through the aryl hydrocarbon receptor-tissue factor axis. Blood. 2019 Dec 26;134(26):2399-2413.

39. Zhang R, Li T, Wang W, Gan W, Lv S, Zeng Z, Hou Y, Yan Z, Yang M. Indoleamine 2, 3-Dioxygenase 1 and $C D 8$ expression profiling revealed an immunological subtype of colon cancer with a poor prognosis. Front Oncol. 2020; 10:594098.

\section{Figures}

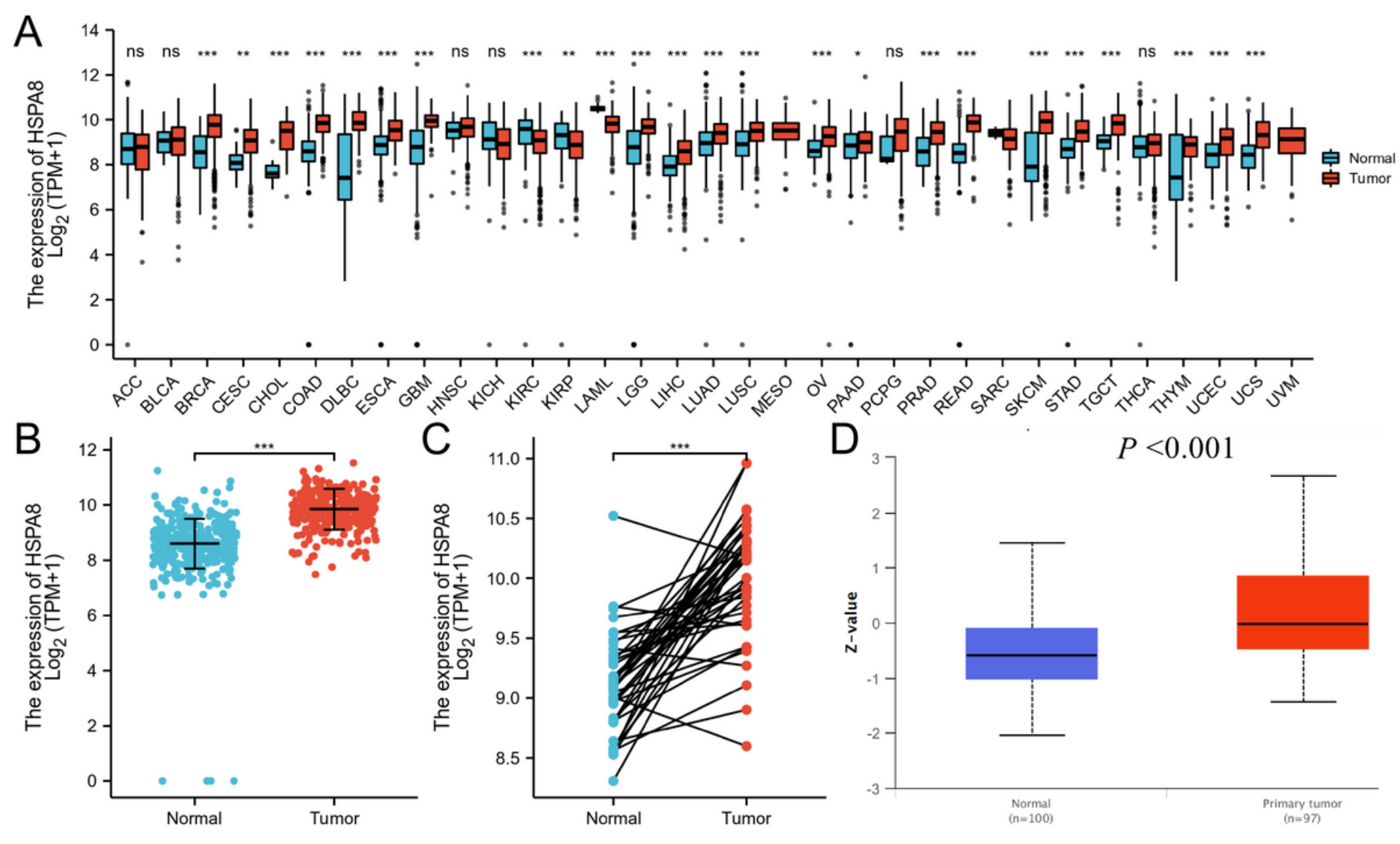

Figure 1 
HSPA8 expression levels were shown in tumor compared with normal tissues. (A) HSPA8 expression in paired pan-cancer and normal samples. (B) HSPA8 expression in unpaired normal and COAD samples (349 vs. 290). (C) HSPA8 expression in paired normal and COAD samples (41 vs. 41). (D) Protein expression of HSPA8 in normal and COAD samples form CPTAC (100 vs.97). NS: $P=0.05$ or higher; *, $\mathrm{P}<0.05 ; * *, \mathrm{P}<0.01 ; * * *, \mathrm{P}<0.001$.
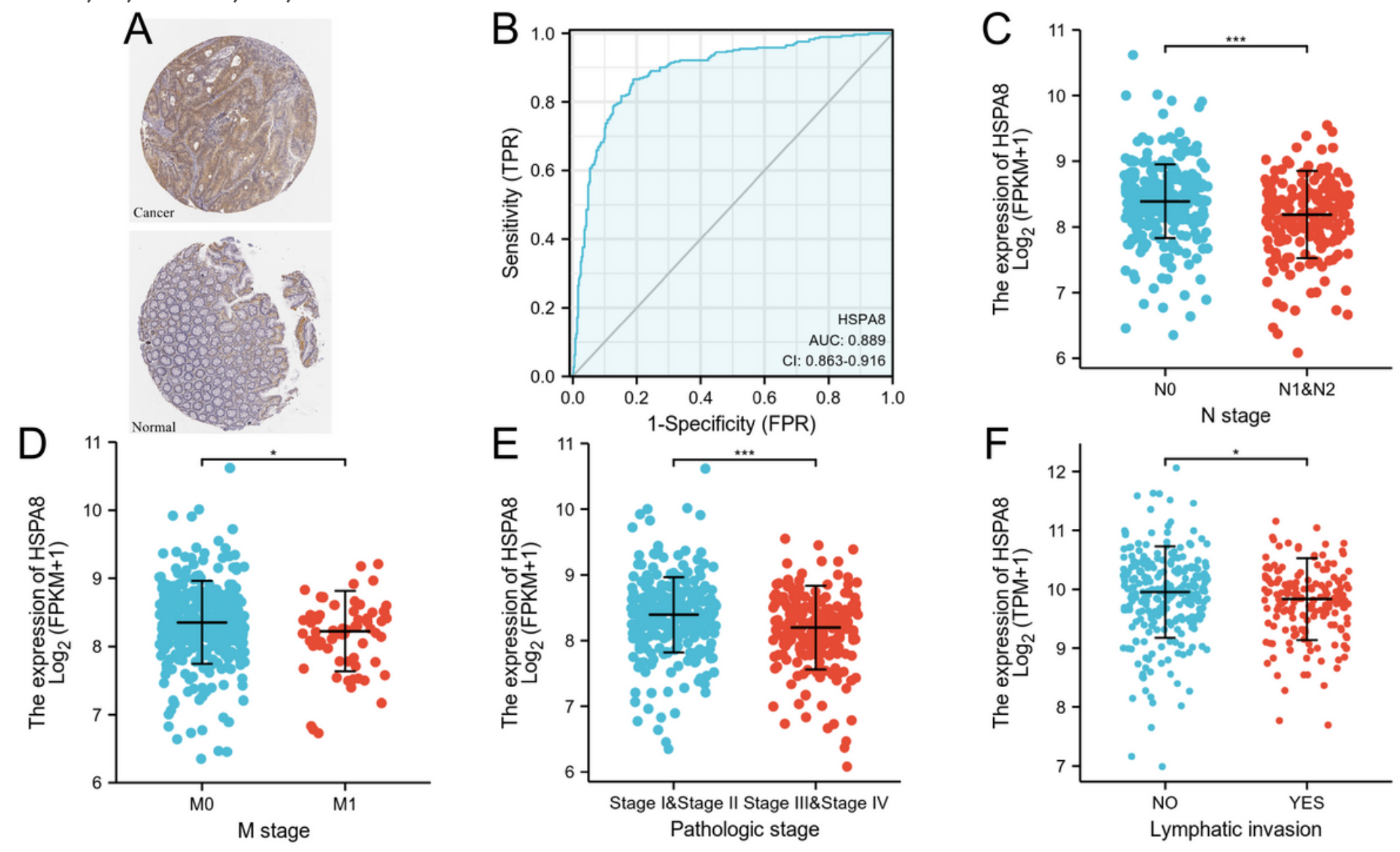

Figure 2

Analysis of the HSPA8 expression in Human Protein Atlas and TCGA (Antibody HPA052504, 10X). (A) HSPA8 protein expression in COAD increased significantly comparing with normal sample. (B) The diagnostic efficacy of HSPA8 in COAD analyzed by ROC (AUC: 0.889). (C-F) Association between HSPA8 expression and $\mathrm{N}$ stage, $\mathrm{M}$ stage, pathologic stage and lymphatic invasion status, the result indicated that HSPA8 decreased significantly with cancer progression. ${ }^{*}, \mathrm{P}<0.05 ;{ }^{* \star *}, \mathrm{P}<0.001$. 

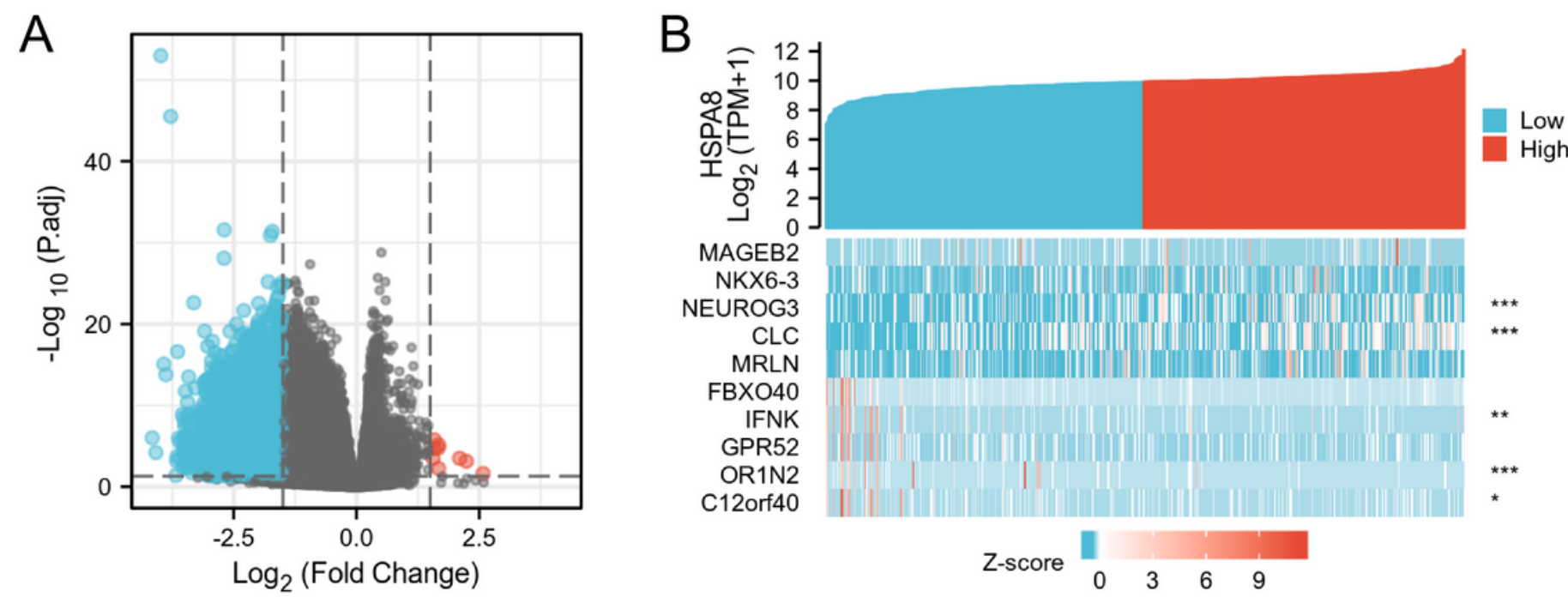

\section{Figure 3}

Differentially expressed genes with high and low HSPA8 expression. (A) Volcano plot of differentially expressed genes between the high and low HSPA8 expression groups. (B) Heatmap of top 10 significantly differentially expressed genes between the high and low HSPA8 expression groups.

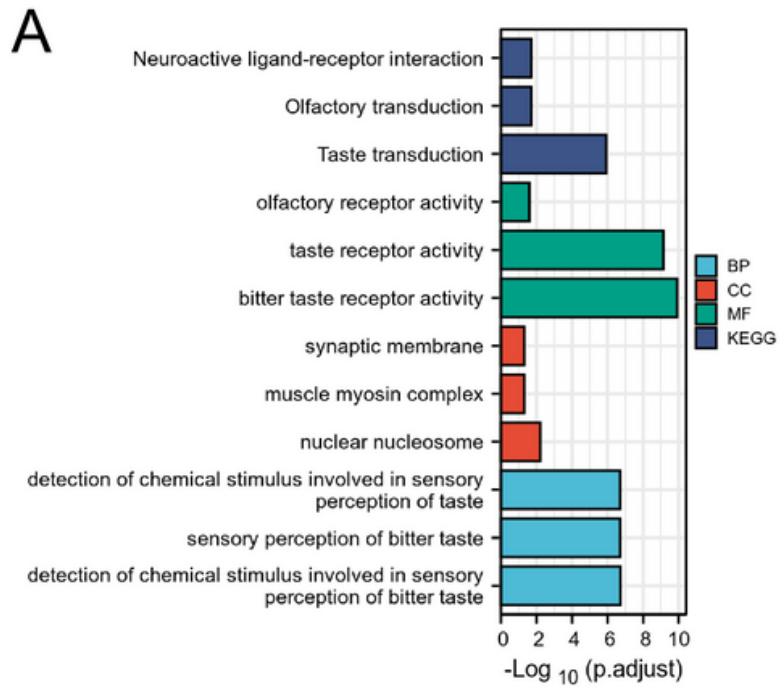

B

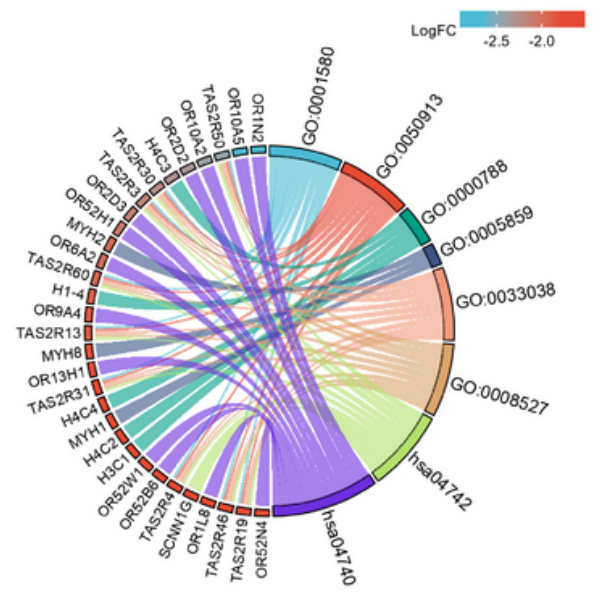

Figure 4

GO/KEGG functional enrichment analysis. (A) The bar graph showed the biological process (BP), cellular component (CC), molecular function (MF) and KEGG of HSPA8. (B) GO chord plot showed the genes are linked via ribbons to their assigned terms. Blue-to-red coding next to the selected genes indicates log FC. 
A

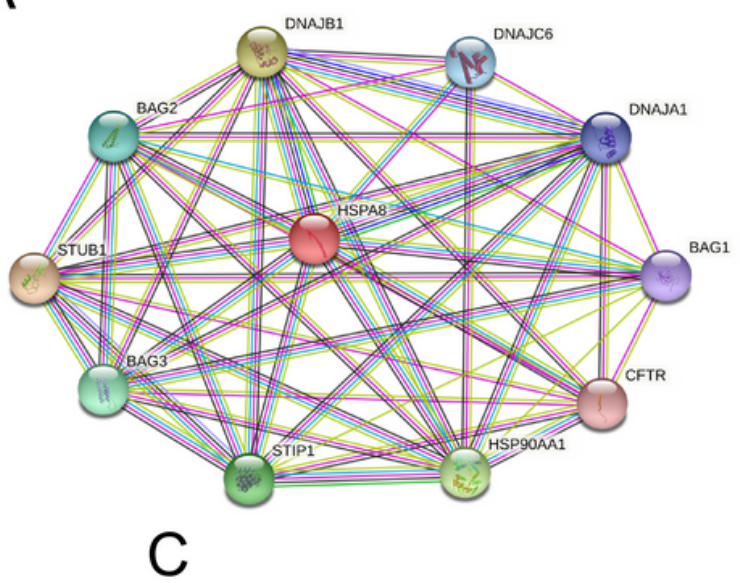

B

\begin{tabular}{ccc} 
Gene symbol & Annotation & Score \\
\hline STUB1 & E3 ubiquitin-protein ligase CHIP & 0.999 \\
DNAJB1 & DnaJ homolog subfamily B member 1 & 0.999 \\
HSP90AA1 & Heat shock protein HSP 90-alpha & 0.999 \\
STIP1 & Stress-induced-phosphoprotein 1 & 0.999 \\
BAG3 & BAG family molecular chaperone regulator 3 & 0.999 \\
BAG2 & BAG family molecular chaperone regulator 2 & 0.999 \\
DNAJC6 & Putative tyrosine-protein phosphatase auxilin & 0.999 \\
DNAJA1 & DnaJ homolog subfamily A member 1 & 0.999 \\
BAG1 & BAG family molecular chaperone regulator 1 & 0.999 \\
CFTR & Cystic fibrosis transmembrane conductance regulator & 0.998 \\
\hline
\end{tabular}

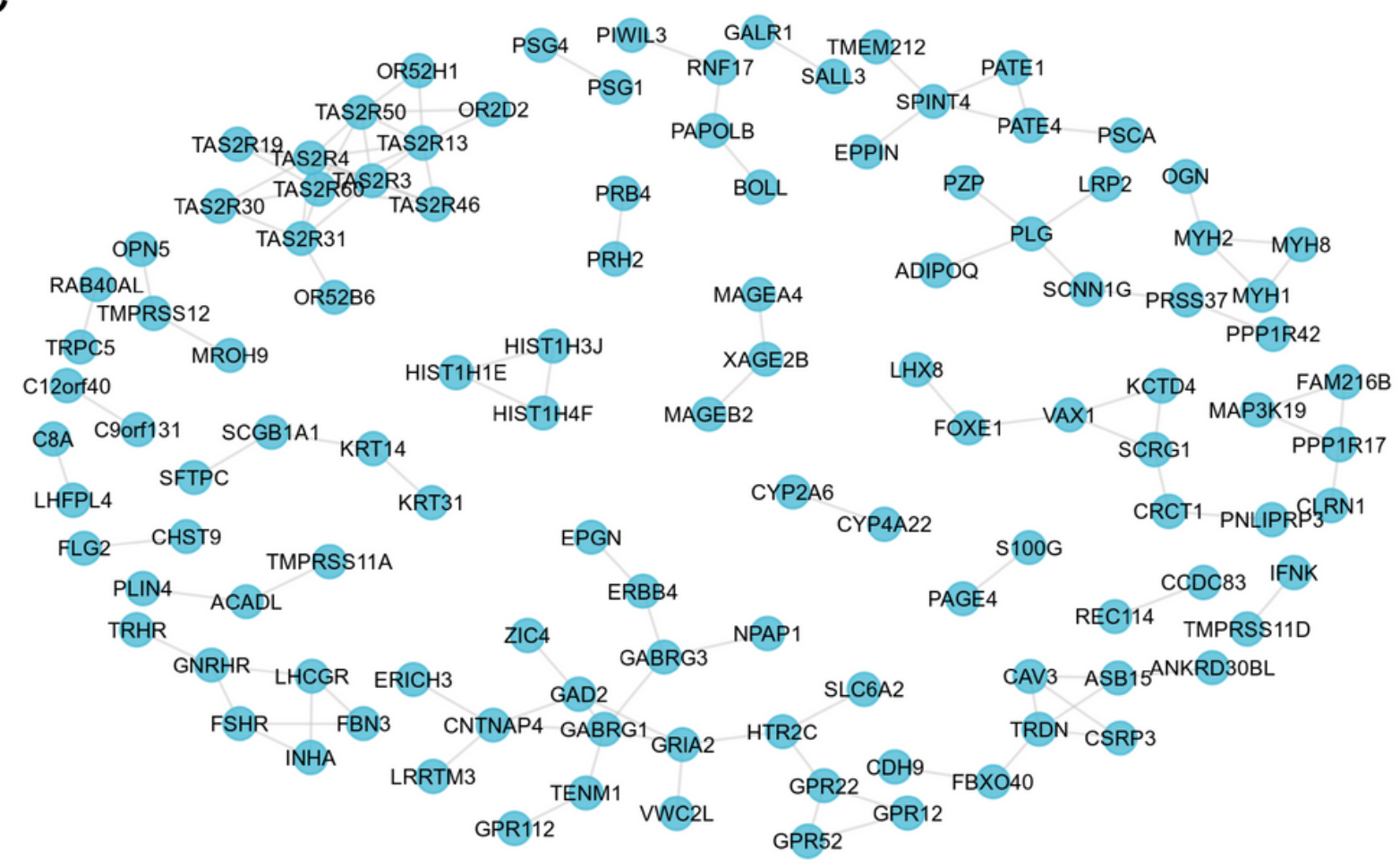

Figure 5

PPI network of HSPA8. (A, C) The network of HSPA8 and its potential co-expression genes was analysis by STRING tool. (B) the detailed information of top ten HSPA8-related genes, as well as their annotations and score were listed. 


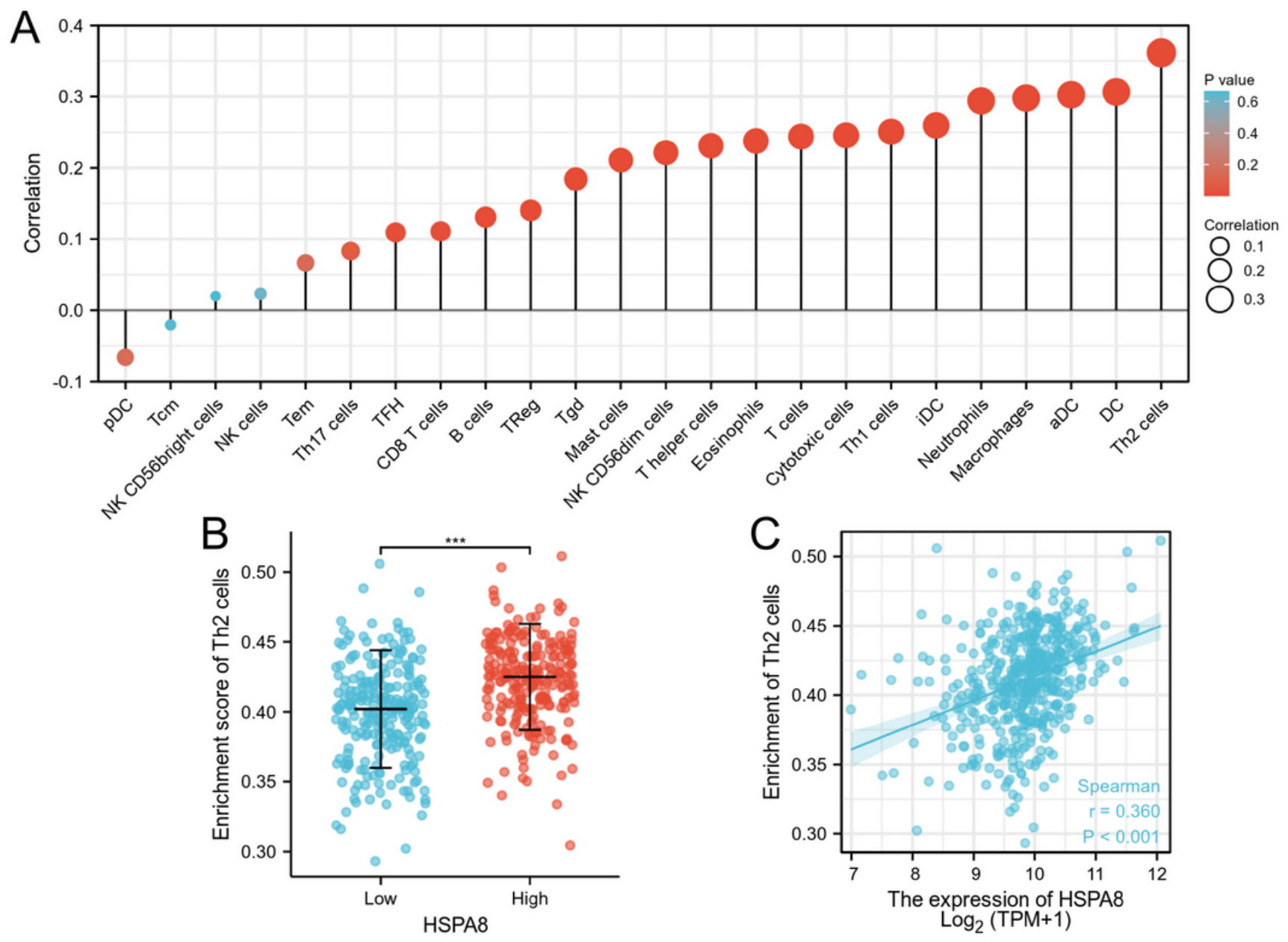

Figure 6

The HSPA8 expression was associated with immune infiltration in the COAD microenvironment. (A) The forest plots showed a positive correlation between HSPA8 and 22 immune cells, and a negative correlation between HSPA8 and 2 immune cell subsets. (B) Infiltration of Th2 between low- and highHSPA8 expression. (C) Correlation between the relative enrichment score of Th2 cells and the expression level of HSPA8. 


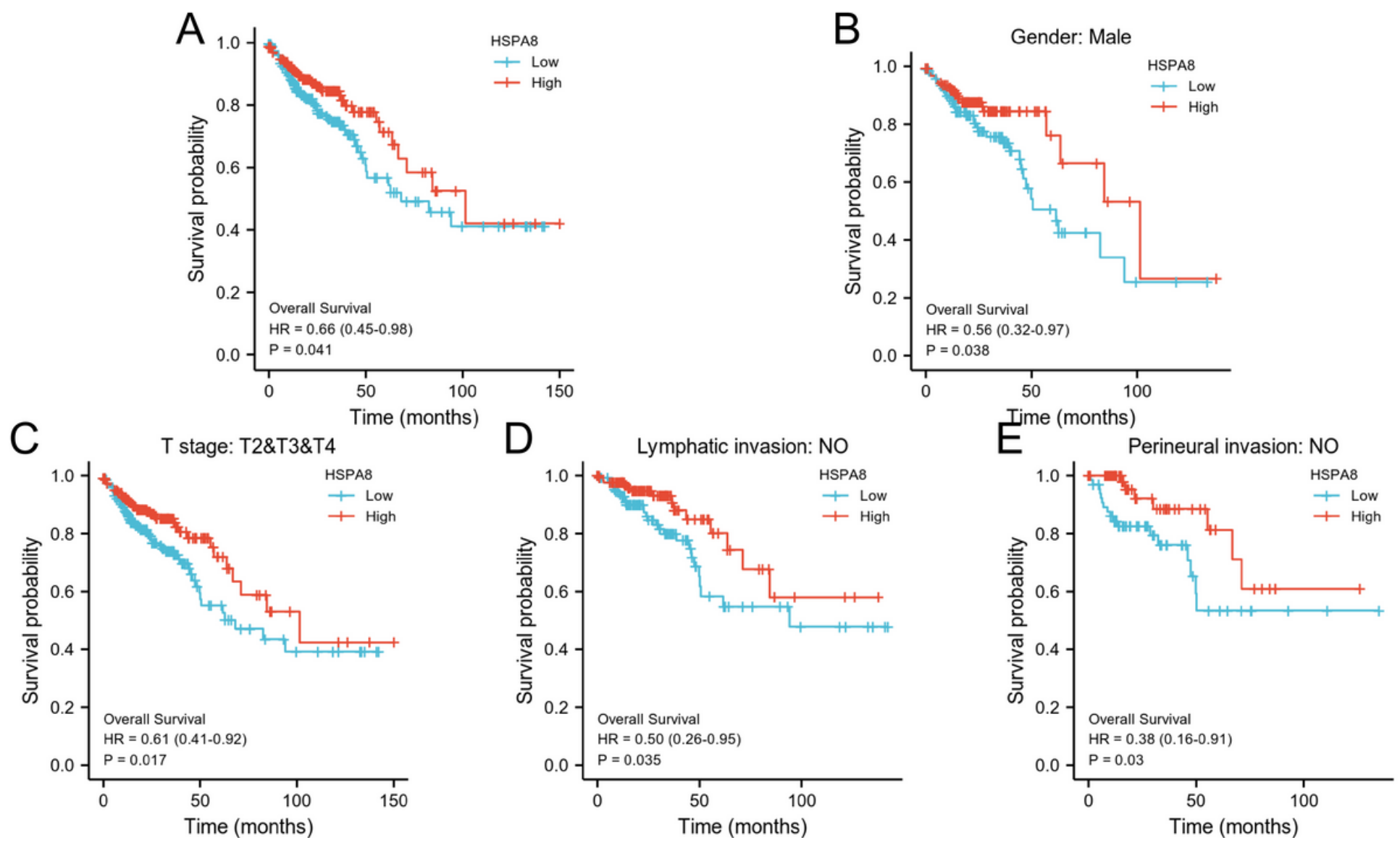

Figure 7

High expression of HSPA8 indicated favorable OS in COAD patients by using Kaplan-Meier survival curves. (A) Curve in all patients. (B-E) Curves in subgroups with male, T2-T4 stage, no lymphatic invasion and no perineural invasion in COAD patients.

\section{Supplementary Files}

This is a list of supplementary files associated with this preprint. Click to download.

- Supplementary1.xlsx

- Supplementary2.xlsx 